
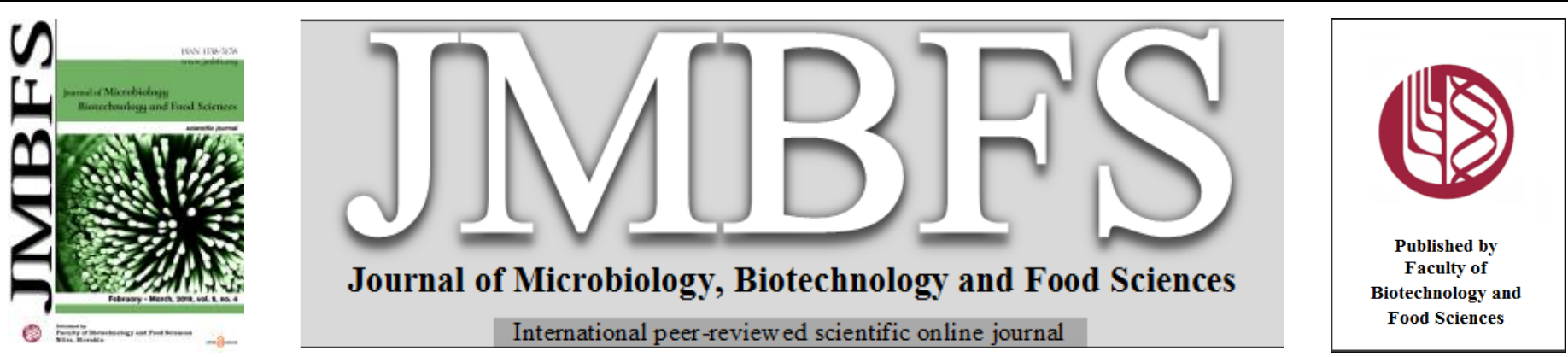

\title{
APPLICATION OF DNA MARKERS FOR THE ASSESSMENT OF ALLELE STATE OF THE KEY GENES OF CAROTENOGENESIS IN MAIZE (ZEA MAYS L.) SEEDS
}

\author{
Larysa Prysiazhniuk*1, Yirii Honcharov $^{2}$, Serhii Melnyk ${ }^{1}$, Iryna Dikhtiar ${ }^{1}$ \\ Address(es): $\mathrm{PhD}$ of of agricultural sciences Larysa Prysiazhniuk, \\ ${ }^{1}$ Ukrainian Institute for Plant Variety Examination, Department of Laboratorial researching on qualifying examination of plant varieties (The Center for Certification \\ Testing), Henerala Rodymtseva Str., 15, 03041, Kyiv, Ukraine, +380442583456 . \\ ${ }^{2}$ Research Institute of Agrarian Business, 80 Berezynska Str., Dnipro, 49130, Ukraine, +380562399100.
}

*Corresponding author: prysiazhniuk_1@ukr.net

doi: 10.15414/jmbfs.2019.8.5.1141-1144

ARTICLE INFO

Received 31. 10. 2018

Revised 28. 11. 2018

Accepted 28. 11. 2018

Published 1. 4. 2019

Regular article

open $\partial_{\text {ACCESS }}$

\begin{abstract}
Presented in the article are the results of the assessment of 108 maize lines in terms of presence of favourable alleles of high carotenoid content in grain. The relationship between the genetic distances of the allele state of the key carotenogenesis genes and the content of carotenoids in maize grain was determined using the Mantel test. Favourable alleles in maize lines were identified by three out of four carotenogenesis markers under study: lcye-SNP216, lcye-3'INDL and $c r t R B 1-3^{\prime} T E$. The frequencies of the favourable alleles varied from 0.28 by markers $l c y \varepsilon-S N P 216$ and $c r t R B 1-3^{\prime} T E$ to 0.90 by marker $l c y \varepsilon-3^{\prime} I N D L$. Eight of the studied lines contained favourable alleles by these polymorphic markers. According to the result of spectrophotometric analysis of the content of carotenoids, the highest value (7.15 $\mu \mathrm{g} / \mathrm{mg}$ ) was found in line DK2732 and the lowest $(0.7 \mu \mathrm{g} / \mathrm{mg})$ in line ISK31. In order to assess the efficiency of the proposed markers for evaluation of the content of carotenoids in maize grain, genetic distances between the studied lines were determined by the allele state of the genes under study and by the content of carotenoids. Mantel test showed that there is a correlation between genetic distances

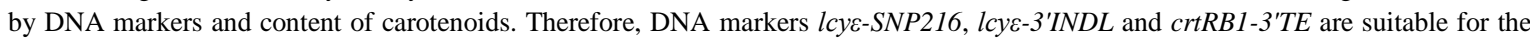
assessment of maize breeding material for the purpose of selection for a high content of carotenoids in grain.
\end{abstract}

\section{INTRODUCTION}

One of the ways to combat vitamin A deficiency in the world is to grow plants that can accumulate carotenoids. Compared with other crops, maize can accumulate a significant amount of carotenoids in seeds during the development of endosperm (Gallager et al., 2004; Adeyemo et al., 2012; Tiwari et al., 2012; Bartley and Scolnic, 1995; Wurtzel, 2004). Biosynthesis of carotenoids occurs before the time of physiological maturation. After that, the concentration of carotenoids changes due to water loss. As a result of a sequence of biochemical reactions, carotenoids and xanthophytes are produced from geranyl-geranyl pyrophosphate, the source material (Muthusamy et al., 2014; Muthusamy et al., 2015; Fu et al., 2013; Zhou et al., 2012).

Today, marker-associated breeding is a topical area for a selection of breeding materials, including those with a high content of $\beta$-carotene in grain. The genes lcye, crtRB1 (Harjes et al., 2008; Yan et al., 2010) and crtRB1 (Xu et al., 2009; Yan et al., 2010; Liu et al. 2015) are considered the main genes affecting the content of carotenoid in maize grain. Harjes et al. (2008) investigated the polymorphism of $l c y \varepsilon$ and identified three polymorphic sites most closely related to the variation in the content of carotenoids. These sites can be considered as molecular markers of the allele state of this gene: an indel at the 5'-end (marker lcye-5 'INDL), a single nucleotide replacement within this gene (marker lcyeSNP216) and an indel at the $3^{\prime}$-end (marker $l c y \varepsilon-3^{\prime} I N D L$ ). For marker $l c y \varepsilon$ $3^{\prime} I N D L$, there are two alleles that have an electrophoregram size of $144+502 \mathrm{bp}$ (favourable allele) and $399+502$ bp (unfavourable allele). For marker lcyzSNP216, there are two possible alleles: 395 bp which corresponds to the presence of a nucleotide with a nitrogen-based thymine ( $\mathrm{T}$, unfavourable allele) in this marker site; the absence of bands on electrophoregram, i.e., zero-alleles that corresponds to the presence of a nucleotide with a nitrogen-based guanine $(\mathrm{G}, \mathrm{a}$ favourable allele) in the marker site. For marker lcye-5'TE, four alleles can be identified: allele $1(150+280 \mathrm{bp})$, allele $2(250 \mathrm{bp})$, allele $3(250 \mathrm{bp}+380 \mathrm{bp})$ and allele $4(650 \mathrm{bp})$. Of these, allele 1 and allele 4 are known as favourable for enhancing the synthesis of carotenoids. crtRB1 is one of the key genes of carotenoid biosynthesis associated with carotenoids accumulation in maize endosperm (Xu et al., 2009; Yan et al., 2010; Liu et al., 2015). There are three polymorphic regions of $c r t R B 1$ that affect the concentration of carotenoids in grain and which are also considered as markers for the allelic state of this gene: a region at the 5'-end, the polymorphism of which is caused by the action of the transposon element (marker $c r t R B 1-5^{\prime} T E$ ); a region within the gene (the marker crtRB1-IND14); the site at the 3'-end, the polymorphism of which is also due to the action of the transposon element (marker crtRB1-3'T) (Sagare et al., 2015; Berman et al., 2017; Zunjare et al., 2018). The polymorphism of $c r t R B 1$ gene by marker crtRB1-3'Te is determined by the appearance of three alleles that form bands at $543 \mathrm{bp}$ on electrophoregram: (allele 1 - favourable allele), $296+875 \mathrm{bp}$ (allele 2) and $296 \mathrm{bp}$ (allele 3). The investigation of the allelic state of these genes makes it possible to detect high- $\beta$-carotene genotypes by polymerase chain reaction (PCR). However, to prove the efficiency of the use of DNA markers, confirmation is required through alternative chemical methods and statistical analysis (Muthusamy et al., 2015; Sagare et al., 2018).

One of the main problems in the statistical processing of data to determine the correlation between the presence of favourable alleles and the content of $\beta$ carotene is the diversity of data. The solution to this problem is the use of alternative methods for determining the correlation, i.e. determination of the correlations between genetic distances by Mantel test, specifically, the definition of regression, where the variables are matrices of similarity which sum up pairs of similar values between the sampling points. The purpose of our research was to assess maize genotypes by the content of carotenoids and to determine the efficiency of using DNA markers in the selection for a high content of carotenoids

\section{MATERIAL AND METHODS}

In the research, 108 inbred maize lines selected at the Institute of Grain Crops National Academy of Agrarian Sciences (Dnipro, Ukraine) were studied. The work was carried out at the Laboratory of Biotechnology of the Institute of Grain Crops (Dnipro, Ukraine) and at the Department of Laboratory Studies on the Qualification Expertise of Plant Varieties (Centre of Certification Tests) of the Ukrainian Institute for Plant Variety Examination (Kyiv, Ukraine) during the 2016-2018 period. 


\section{DNA isolation and PCR}

DNA was isolated from $100 \mathrm{mg}$ of 5-day sprouts using CTAB and dissolved in TE buffer (Velikov, 2013). Three functional markers for lcye (Harjes $\boldsymbol{e t}$ al. 2008; Muthusamy et al., 2014) and one marker for crtRB1 was used (Muthusamy et al. 2015) (Tab 1)

Table 1 Functional markers and primer sequences to them

\begin{tabular}{|c|c|}
\hline Marker name & $\begin{array}{l}\text { The nucleotide sequence of primers } \\
5, \rightarrow 3\end{array}$ \\
\hline lсye-5'TE & $\begin{array}{l}\text { F aag cat ccg acc aaa ata aca } g \\
R \text { gag agg gag acg acg aga cac }\end{array}$ \\
\hline$l$ cye-SNP216 & $\begin{array}{c}\text { F gcg gca gtg ggc gtg gat } \\
\mathrm{R} \text { tga agt acg gct gca gga caa cg }\end{array}$ \\
\hline \multirow{2}{*}{$l c y \varepsilon-3$ 'INDL } & $\begin{array}{l}\text { F1 gta cgt cgt tca tct ccg tac cc } \\
\text { R1 ctt ggt gaa cgc att tct gtt gg }\end{array}$ \\
\hline & $\begin{array}{l}\text { F2 gga ccg gaa cag cca act g } \\
\text { R2 ggc gaa atg ggt acg gcc) }\end{array}$ \\
\hline crtRB1-3'TE & $\begin{array}{l}\text { F aca cca cat gga caa gtt cg } \\
\text { R1 aca ctc tgg ccc atg aac ac } \\
\text { R2 aca gca ata cag ggg acc ag }\end{array}$ \\
\hline
\end{tabular}

In order identify the single-nucleotide polymorphism by the marker lcysSNP216, an internal control of the reaction was used - the alcohol dehydrogenase gene (adh1), that have an electrophoregram size of 234 п.н. Thus, in the case of identification of a favourable allele, which corresponds to the presence of a nucleotide with a nitrogen-based $\mathrm{G}$ in the marker site, only one allele $234 \mathrm{bp}$ was identifiyв on the electrophoregram. A reaction mixture of $20 \mu$ l contained: $1 \times$ DreamTaq ${ }^{\mathrm{TM}}$ Greenbuffer, $1 \mathrm{u}$ DreamTaq ${ }^{\mathrm{TM}}$ polymerase (ThermoScientific), $200 \mu \mathrm{M}$ of each dNTP, $30 \mathrm{ng}$ of DNA sample, $0.2 \mu \mathrm{M}$ of each primer according to the marker. Polymerase chain reaction (PCR) was carried out on the TC-Y CreaCon (USA) amplifier. For the all primers, the following parameters of PCR were set: step 1 - initial denaturation: $\left(94^{\circ} \mathrm{C}\right) 5 \mathrm{~min}$; step $2-$ development of specific reaction products: denaturation: $\left(94^{\circ} \mathrm{C}\right) 1 \mathrm{~min}$, hybridization of primers $\left(60^{\circ} \mathrm{C}\right) 1 \mathrm{~min}$, elongation $\left(72^{\circ} \mathrm{C}\right) 1 \mathrm{~min}$, number of cycles 30 ; step 3 - final elongation: $\left(72^{\circ} \mathrm{C}\right) 5 \mathrm{~min}$.

Visualization of the amplification products after electrophoretic separation in a $2 \%$ agarose gel was carried out using GelDocTM EZ (U.K.) visualization device. The analysis of obtained electrophoresis was performed on the software Image $\mathrm{Lab}^{\mathrm{TM}}$ Software. Genetic distances and frequencies of detected alleles were calculated based on the determined size of alleles.

Evaluation of the content of carotenoids by the method of spectrophotometry

The content of carotenoids in the samples of maize grain stored for one year prior to the analysis was measured in chloroform spectra using the Rodriguez-Amaya and Kimura (2004) method. Extraction of carotenoids was carried out with chloroform in $0.5 \mathrm{~g}$ samples. The optical density was measured at a wavelength of $450 \mathrm{~nm}$ on the spectrophotometer SF 2000 (Russian Federation) using chloroform as a reference.

\section{Statistical analysis of data}

Genetic distances between the genotypes under study were determined with the aid of cluster analysis using a computer program STATISTICA 12 (Trial version). The grouping of maize lines based on the results of molecular genetic analysis and the content of carotenoids in the grain was carried out using the method of unweighted pair-group avarage with the calculation of Euclidian distances (Fortin et al., 2014; Drozdov, 2010; Dong et al., 2014).

Determination of correlation of maize genotypes polymorphism based on the data molecular genetic analysis and analysis of the content of carotenoids in maize grain was carried out on the basis of their genetic distances using Mantel test and XLSTAT 2018 Software (Trial version) (Legendre et al., 2010; Diniz-Filho et al., 2013)

\section{RESULTS AND DISCUSSION}

\section{Determination of the allelic state of a locus}

As a result of the research, the key molecular genetic markers related to the accumulation of carotenoids in maize seeds were analysed and the content of total carotenoids in the seeds was determined. The state of two key genetic carcinogenesis genes in maize, namely lycopene epsilon cyclase and $\beta$-carotene diroxylase1, was analysed. Polymorphism of the first gene was investigated for three polymorphic markers lcye-5'TE, lcye-SNP216 and lcye-3'INDL, and the polymorphism of the second gene for one marker crtRB1-3'TE.

The polymorphism in the lines under study was detected for three markers (lcye3'INDL, lcye-SNP216 and crtRB1-3'TE); no polymorphism was detected for marker lcye-5'TE.
The frequency of favourable alleles by the markers ranged from 0.28 (lcyeSNP216 and crtRB1-3'TE) to 0.90 (lcye-3'INDL). Given in Table 2 are the summarized research results.

Table 2 Characteristics of the studied loci

\begin{tabular}{|c|c|c|c|}
\hline Marker & Allele & $\begin{array}{c}\text { The total } \\
\text { number of } \\
\text { lines }\end{array}$ & $\begin{array}{c}\text { Allele } \\
\text { frequency }\end{array}$ \\
\hline lсye -5'TE & $250 \mathrm{bp}$ & 108 & 1.00 \\
\hline \multirow{2}{*}{ lсyє -3'INDL } & $144+502$ bp* & 97 & 0.90 \\
\hline & $399+502$ bp & 11 & 0.10 \\
\hline \multirow{2}{*}{ lсye -SNP216 } & $\mathrm{T}$ & 78 & 0.72 \\
\hline & $\mathrm{G}^{*}$ & 30 & 0.28 \\
\hline \multirow{3}{*}{ crtRB1-3'TE } & $543 \mathrm{bp}^{*}$ & 30 & 0.28 \\
\hline & $296+875$ bp & 41 & 0.38 \\
\hline & $296 \mathrm{bp}$ & 37 & 0.34 \\
\hline
\end{tabular}

Legend: *favourable allele

Of 108 maize genotypes under study, 8 lines (7\%) (DK206A, DK212, DK1855, DKD9066, ISK2, ISK3, ISK12 and ISK21) contained favourable alleles by all the markers; 29 lines $(27 \%)$ contained favourable alleles by two markers lcyeSNP216 and lcye-3'INDL; 26 lines (24\%) had favourable alleles by two markers lsye-3'INDL and crtRB1-3'TE; 10 lines $(9 \%)$ had a favourable allele detected by two markers lcye-SNP216 and crtRB1-3'TE. No favourable alleles were found in four maize lines.

Mathusamy et al., (2015) conducted research to identify favourable alleles of high $\beta$-carotene content in maize grain of elite inbred parent components of agronomic hybrids through marker-associated selection. Having analysed 385 maize lines Mathusamy et al. (2015) identified a favourable allele of 650 bp by marker lcye-5'TE in 13 lines and an unfavourable allele of $250 \mathrm{bp}$ in 372 lines. By marker crtRB1-3'TE, a favourable allele of 543 bp was identified in 15 lines, and unfavourable alleles of $296+875 \mathrm{bp}$ and $296 \mathrm{bp}$ in 51 and 319 maize lines, respectively. Noticeably, no line with a favourable allele of $650 \mathrm{bp}$ was detected by marker lcye-5'TE. However, the number of genotypes identified by crtRB13 'TE marker significantly exceeded (28\% and $3 \%$, respectively) the number of lines found by Mathusamy et al. (2015).

Azmach et al. (2013) examined 130 tropical-adapted lines of yellow maize. The frequency of favourable alleles detected by Azmach et al. (2013) varied by a marker with a range of $12-83 \%$. The largest number of lines with a favourable allele was identified by lcye-SNP216 marker. In our study, the frequency of genotypes with a favourable allele by this marker did not exceed $28 \%$. However, it is of importance to note that the results of our research, as well as the results obtained by Azmach et al. (2013), indicate a low frequency of the favourable allele by marker lcye-5'TE. The frequency of a favourable allele of $650 \mathrm{bp}$ in the research carried out by Azmach et al. (2013) was estimated at 12\%; in our research, this allele was not identified at all. The largest number of lines containing favourable alleles was identified by marker lcye-3'INDL, that is $90 \%$ of the total number of the lines under study. Aimed at the evaluation of the total carotenoid content, Azmach et al. (2013) found that the combination of favourable alleles by the two genes, lcye and crtRB1, explains 38 and $89 \%$, respectively, of the total variation of carotenoid content.

\section{Assessment of maize genotypes in terms of the content of carotenoids}

The highest concentration of carotenoids $(7.15 \mu \mathrm{g} / \mathrm{mg})$ was found in line DK2732 and the lowest $(0.7 \mu \mathrm{g} / \mathrm{mg})$ in line ISK31. Our data, i.e. a very wide range of carotenoid content in maize grain, come to an agreement with the results of other researchers.

To illustrate, Belarusian researchers evaluated the content of carotenoids in grain of 22 Belarusian maize lines using the method of spectrophotometry (Orlovskaya et al., 2016). The content of carotenoids amounted to 0.21-1.16 $\mu \mathrm{g} / \mathrm{mg}$. Fu et al. (2013) conducted a quantitative analysis of carotenoids in 129 maize samples and found a wide range of variability by the 'the content of total carotenoids' sign with a range of $1.09-31.89 \mu \mathrm{g} / \mathrm{mg}$. In addition, the researchers pointed out a high index of the sign inheritance $(0.91)$.

The content of carotenoids in grain of the maize lines containing favourable alleles by all three markers was $2.23 \mu \mathrm{g} / \mathrm{mg}$, i.e. significantly exceeded the mean value $(1.95 \mu \mathrm{g} / \mathrm{mg})$. The content of carotenoids in those maize lines that did not contain favourable alleles by all the markers under study was estimated at 1.71 $\mu \mathrm{g} / \mathrm{mg}$.

\section{Statistical analysis}

In order to evaluate the polymorphism of the key carotenogenesis genes and the content of carotenoid in grain of maize lines, we conducted a cluster analysis and calculated genetic distances between genotypes.

The results of the cluster analysis on the state of the key carotenogenesis genes show that the most distant are those lines that have the value of genetic distances 2.45. The least value of genetic distances found among pairs of genotypes was 0.00 . In other cases, the values of genetic distances between objects of the 
sampling were 1.41 and 2.00. Consequently, taking into account that a fact of presence/absence of favourable alleles relates to the accumulation of carotenoids in maize seeds, the genotypes under study were divided into four groups according to genetic distances between individual genotypes. Given that identical objects are those with a digital expression of genetic distances equal to ' 0 ', we can conclude that such lines do not differ from each other.

According to the data of the cluster analysis on the content of carotenoids in maize grain, a high variability of maize lines distribution was found. Taking into account that the initial data of the cluster analysis on the content of carotenoids were represented by an array of absolute values resulted from the spectrophotometric analysis, the value of genetic distances between the lines varies from 0.1 to 18.5 . Here it should be noted that lines DK2732, DK6356 and DK2323 are the most distant from the other lines under study. Thus, the value of genetic distances between line DK2732 and the others averages 15.8. For lines DK6356 and DK2323, the average genetic distances were 10.0 and 8.9, respectively. As noted above, line DK2732 has the highest value of the content of carotenoids. Lines DK6356 and DK232 also have a high content of carotenoids (5.23 and $4.86 \mu \mathrm{g} / \mathrm{mg}$, respectively). It is important to note that lines DK2732 and DK2323 have favourable alleles by two markers (lcye-3'INDL and lcyeSNP216), and line DK6356 line by only one (lcye-3'INDL). Considering the results of the research, it is possible to assume that markers lcye-3'INDL and lcye-SNP216 are the most efficient for selection. This can be explained by the fact that these markers are the most significantly related to the variation in the content of carotenoids in grain (Harjes et al., 2008; Xu et al., Yan et al., 2010 Liu et al. 2015)

To assess the correlation of genetic distances between maize lines and the conten of carotenoids in their seeds Mantel test (Pearson linear correlation) was used.

As a result of the assessment, $\mathrm{p}$-value and $\mathrm{r}(\mathrm{AB})$ values at the significance level $\alpha=0.05$ were determined, which, according to the interpretation of the test results, allows one of the hypotheses for the presence $(\mathrm{Ha})$ or no correlation $(\mathrm{H} 0)$ to be made (Figure 1)

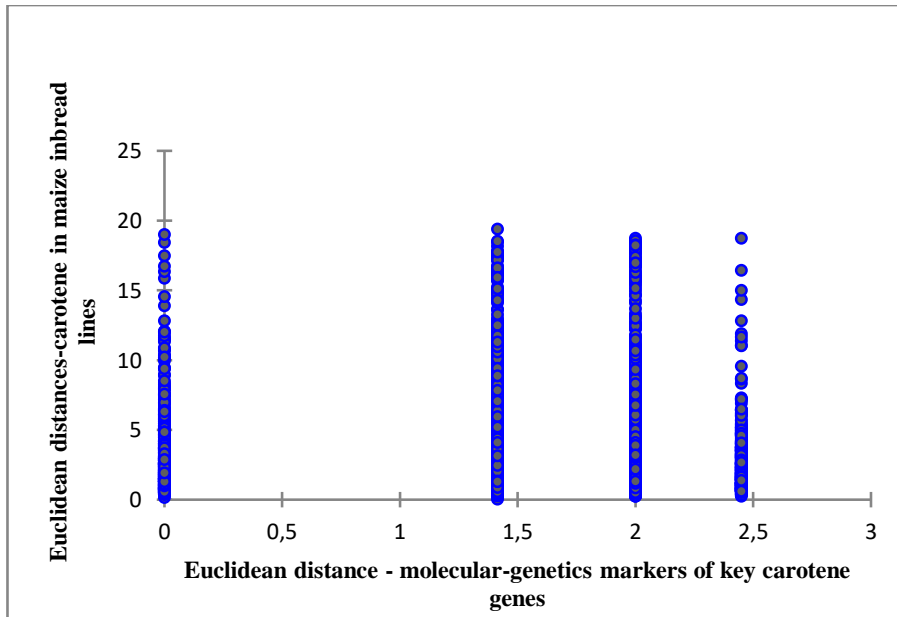

Figure 1 The relationship between genetic distances of maize genotypes by fou markers of carotenogenesis and the content of carotenoids

It is known that the hypothesis $\mathrm{H} 0$ of the absence of correlation can be assumed given that $p>\alpha$. As a result of our research, the calculated value of $p(0.021)$ was lower than the significance level $\alpha=0.05$, therefore, it is necessary to reject the 'zero' hypothesis H0 and adopt the alternative hypothesis Ha (Burstin et al. 1997; Diniz-Filho et al., 2013). Shown in Figure 2 is the distribution of the data by matrices of genetic distances and the correlation coefficient.

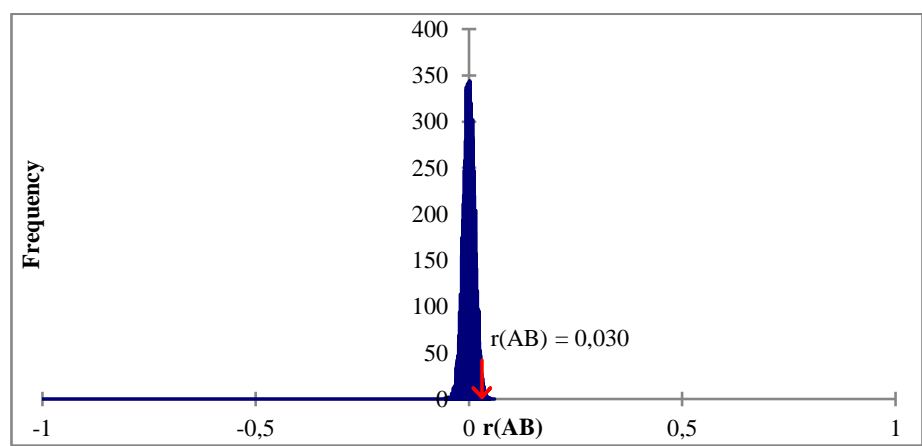

Figure 2 Graphical analysis of the data distribution according to the results of the Mantel test by genetic distances

On the basis of the analysis, the correlation between genetic distances of 108 maize genotypes identified by four markers for the key carotenogenesis genes and the content of carotenoids in maize grain was found. Because by the marker $l c y \varepsilon-5^{\prime} T E$ any favorable alleles have not been detected, it can be assumed that the correlation coefficient between genetic distances of maize lines will have a different value or the corelation will be absent. In order to verify this statement, we analyzed genetic distances between content of carotenoids in grain and three DNA markers (without marker $l$ cye -5 'TE). The obtained data also show the presence of correlation. However, it should be noted that the correlation coefficient is 0.023 . It can be explained by the fact that the calculation of genetic distances by the unweighted pair-group avarage method provides for determining the level of closeness between the objects of analysis both by the presence of a certain criterion and by its absence. It was revealed that favourable for a high content of carotene in grain alleles were identified by three out of four markers under investigation. It was observed that the lines which according to the results of the spectrophotometric analysis demonstrated the highest values of the content of carotenoids had favourable alleles by at least one marker (lcye-3'INDL). The highest values of the content of carotenoids were found in line DK2732 which had favourable alleles by two markers, namely $l c y \varepsilon-3$ 'INDL and $l c y \varepsilon-S N P 216$. Therefore, three markers under study, namely $l c y \varepsilon-3 ' I N D L, l c y \varepsilon-S N P 216$ and crtRB1-3'TE shall be recommended for using in the selection of maize lines as genetic material that has favourable alleles for a high content of carotenoids in grain.

\section{CONCLUSIONS}

Molecular and genetic polymorphism of 108 maize lines was investigated by four key markers of carotenogenesis. The content of carotenoids in maize grain lines was determined.

Maize genotypes containing favourable alleles by the investigated markers were identified based on the molecular and genetic analysis of the carotenogenesis markers. Thus, 30 maize lines have a favourable allele by markers $l c y \varepsilon-S N P 216$ and $c r t R B 1-3$ 'TE and 97 lines by marker $l c y \varepsilon-3$ 'INDL. The content of carotenoids in maize grain varied by genotype ranging from 0.70 to $7.15 \mu \mathrm{g} / \mathrm{mg}$.

Based on the results of the Mantel test, there is a correlation between the genetic distances identified by carotenogenesis markers and the content of carotene in maize grain.

In conclusion, it is advisable to use the proposed markers in the selection for a high content of carotene in maize grain. The selected lines, which have favourable alleles based on the state of the key carotenogenesis genes, will be used for the production of hybrids.

\section{REFERENCES}

Adeyemo, O., Menkir, A., Melaku, G., \& Omidiji, O. (2012). Genetic diversity assessment andrelationship among tropicalyellow endosperm maize inbred lines using SSR markers. Maydica, 56(1), 1-7.

Azmach, G., Gedil, M., Menkir, A., \& Spillane, C. (2013). Marker-trait association analysis of functional gene markers for provitamin A levels across diverse tropical yellow maize inbred lines. BMC plant biology, 13(1), 227 https://doi.org/10.1186/1471-2229-13-227

Bartley, G. E., \& Scolnik, P. A. (1995). Plant carotenoids: pigments for photoprotection, visual attraction, and human health. The Plant Cell, 7(7), 1027. http://dx.doi.org/10.1105/tcp.7.7.1027.

Berman, J., Zorrilla-López, U., Sandmann, G., Capell, T., Christou, P., \& Zhu, C. (2017). The Silencing of Carotenoid $\beta$-Hydroxylases by RNA Interference in Different Maize Genetic Backgrounds Increases the $\beta$-Carotene Content of the Endosperm. International journal of molecular sciences, 18(12), 2515. https://doi:10.3390/ijms18122515.

Burstin, J., Charcosset, A. (1997). Relationship between phenotypic and marker distances: theoretical and experimental investigations. Heredity, 79(5): 477.

Diniz-Filho, J. A. F., Soares, T. N., Lima, J. S., Dobrovolski, R., Landeiro, V. L. Telles, M. P. D. C., ... \& Bini, L. M. (2013). Mantel test in population genetics. Genetics and molecular biology, 36(4), 475-485. http://dx.doi.org/10.1590/S1415-47572013000400002.

Dong, D., Fu, X., Yuan, F., Chen, P., Zhu, S., Li, B., ... \& Zhu, D. (2014). Genetic diversity and population structure of vegetable soybean (Glycine max (L.) Merr.) in China as revealed by SSR markers. Genetic resources and crop evolution, 61(1), 173-183. http://doi:10.1007/s10722-013-0024-y.

Drozdov, V. I. (2010). Instructions for using the Statistica 6.0. Kursk: YuZGU. (in Russian).

Fortin, M. J., Dale, M. R., \& Ver Hoef, J. M. (2014). Spatial analysis in ecology. Wiley StatsRef: Statistics Reference Online, 1-13. http://doi:10.1002/9781118445112.stat07766.

http://onlinelibrary.wiley.com/doi/10.1002/9781118445112.stat07766.pub2/full Fu, Z., Chai, Y., Zhou, Y., Yang, X., Warburton, M. L., Xu, S., ... \& Yan, J. (2013). Natural variation in the sequence of PSY1 and frequency of favorable polymorphisms among tropical and temperate maize germplasm. Theoretical and applied genetics, 126(4), 923-935. https://doi:10.1007/s00122-012-2026-0.

Gallagher, C. E., Matthews, P. D., Li, F., \& Wurtzel, E. T. (2004). Gene duplication in the carotenoid biosynthetic pathway preceded evolution of the grasses. Plant Physiology, 135(3), 1776-1783. 
Harjes, C. E., Rocheford, T. R., Bai, L., Brutnell, T. P., Kandianis, C. B., Sowinski, S. G., ... \& Yan, J. (2008). Natural genetic variation in lycopene epsilon cyclase tapped for maize biofortification. Science, 319(5861), 330-333. http://doi:10.1126/science.1150255

Legendre, P., \& Fortin, M. J. (2010). Comparison of the Mantel test and alternative approaches for detecting complex multivariate relationships in the spatial analysis of genetic data. Molecular ecology resources, 10(5), 831-844 https://doi:10.1111/j.1755-0998.2010.02866.x.

Liu, L., Jeffers, D., Zhang, Y., Ding, M., Chen, W., Kang, M. S., \& Fan, X. (2015). Introgression of the crtRB1 gene into quality protein maize inbred lines using molecular markers. Molecular breeding, 35(8), 154 http://doi:10.1007/s11032-015-0349-7.

Muthusamy, V., Hossain, F., Thirunavukkarasu, N., Choudhary, M., Saha, S. Bhat, J. S., ... \& Gupta, H. S. (2014). Development of $\beta$-carotene rich maize hybrids through marker-assisted introgression of $\beta$-carotene hydroxylase allele. PLoS One, 9(12), e113583. https://doi:10.1371/journal.pone. 0113583.

Muthusamy, V., Hossain, F., Thirunavukkarasu, N., Saha, S., \& Gupta, H. S (2015). Allelic variations for lycopene- $\varepsilon$-cyclase and $\beta$-carotene hydroxylase genes in maize inbreds and their utilization in $\beta$-carotene enrichment programme. Cogent Food \& Agriculture, 1(1), 1033141. https://doi.org/10.1080/23311932.2015.1033141.

Orlovskaya, O. A., Vakula, S. I., Khotyleva, L. V., \& Kilchevsky, A. V. (2016) Assotiation of total carotenoid level in maize grain (Zea mays L.) with polimorphic site InDel1 in PSY1 gene. Ecological genetics, 14(3), 28-34 http://doi.org/10.17816/ecogen14328-34

Rodriguez-Amaya, D. B., \& Kimura, M. (2004). HarvestPlus handbook for carotenoid analysis (Vol. 2). Washington, DC: International Food Policy Research Institute (IFPRI).

Sagare, D. B., Shetti, P., Reddy, S. S., Surender, M., \& Pradeep, T. (2015) Screening of maize (Zea mays L.) germplasm for crtRB1-3'TE allele enhancing provitamin A concentration in endosperm. Res. Environ. Life Sci, 8(4), 673-674.

Sagare, D. B., Shetti, P., Surender, M., Reddy, S. S., Pradeep, T., \& Anuradha, G. (2018). Maize: potential crop for provitamin A biofortification. Maydica, 63(2), 11.

Tiwari, A., Prasanna, B. M., Hossain, F., \& Guruprasad, K. N. (2012). Analysis of genetic variability for kernel carotenoid concentration in selected maize inbred lines. Indian Journal of Genetics and Plant Breeding, 72(1), 1.

Velikov, V. A. (2013). Molecular biology. A Practical Guide. Saratov: Saratovskiy istochnik. (in Russian)

Wurtzel, E. T. (2004). Chapter five Genomics, genetics, and biochemistry of maize carotenoid biosynthesis. In Recent advances in phytochemistry (Vol. 38 pp. 85-110). Elsevier.

Xu, Y., Skinner, D. J., Wu, H., Palacios-Rojas, N., Araus, J. L., Yan, J., ... \& Crouch, J. H. (2009). Advances in maize genomics and their value for enhancing genetic gains from breeding. International journal of plant genomics, 2009. http://doi:10.1155/2009/957602.

Yan, J., Kandianis, C. B., Harjes, C. E., Bai, L., Kim, E. H., Yang, X., ... \& Fernandez, M. G. S. (2010). Rare genetic variation at Zea mays crtRB1 increases $\beta$-carotene in maize grain. Nature genetics, 42(4), 322. http://doi:10.1038/ng.551. Zhou, Y., Han, Y., Li, Z., Fu, Y., Fu, Z., Xu, S., .. \& \& Yang, X. (2012). ZmcrtRB3 Encodes a Carotenoid Hydroxylase that Affects the Accumulation of $\alpha$-carotene in Maize Kernel F. Journal of integrative plant biology, 54(4), 260269. https://doi:10.1111/j.1744-7909.2012.01106.x

Zunjare, R. U., Hossain, F., Muthusamy, V., Baveja, A., Chauhan, H. S., Bhat, J. S., ... \& Gupta, H. S. (2018). Development of Biofortified Maize Hybrids through Marker-Assisted Stacking of $\beta$-Carotene Hydroxylase, Lycopene- $\varepsilon$-Cyclase and

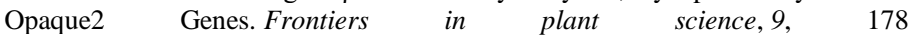
https://doi.org/10.3389/fpls.2018.00178 